\title{
Energy Efficient Clustering and Cluster Head Rotation Scheme for Wireless Sensor Networks
}

\author{
Ashok Kumar, Vinod Kumar \\ Department of E\&CE \\ National Institute of Technology \\ Hamirpur (HP) - INDIA
}

\author{
Narottam Chand \\ Department of CS\&E \\ National Institute of Technology \\ Hamirpur (HP) - INDIA
}

\begin{abstract}
Wireless sensor nodes are highly energy constrained devices. They have limited battery life due to various constraints of sensor nodes such as size and cost, etc. Moreover, most of the Wireless Sensor Network (WSN) applications render it impossible to charge or replace the battery of sensor nodes. Therefore, optimal use of node energy is a major challenge in wireless sensor networks. Clustering of sensor nodes is an effective method to use the node energy optimally and prolong the lifetime of energy constrained wireless sensor network. In this paper, we propose a location based protocol for WSN- supporting an energy efficient clustering, cluster head selection/rotation and data routing method to prolong the lifetime of sensor network. Proposed clustering protocol ensures balanced size cluster formation within the sensing field with least number of transmitreceive operations. Cluster head rotation protocol ensures balanced dissipation of node energy despite the non-uniform energy requirements of cluster head and sensor nodes in a cluster. The cluster head rotation protocol has been proposed to achieve the balanced energy consumption among the nodes within the cluster thus prolonging the lifetime of network Simulation results demonstrate that proposed protocol prolongs network lifetime due to the use of efficient clustering, cluster head selection/rotation and data routing.
\end{abstract}

Keywords- Corona; clusters;cluster head; sink; network lifetime.

\section{INTRODUCTION}

Recent advancements in large scale integration and wireless communication technologies have enabled the development of small size, low cost, and multi-functional devices known as sensor nodes. Sensor nodes are capable of sensing the desired environmental parameters within their vicinity, such as temperature, pressure, moisture, and pollutants, etc., converting the sensed variable to electrical signal and transmitting the sensed data to the desired destination. To achieve these objectives, sensor nodes are equipped with sensors, microcontrollers/microprocessors, and wireless trans-receivers. When these sensor nodes are deployed in large number to monitor an area, they form self-organizing cooperative wireless ad-hoc network, known as wireless sensor network. The wireless sensor networks are mostly deployed in remote and hazardous locations, where manual monitoring is very difficult or almost impossible. Due to deployment of wireless sensors in unattended harsh environment, it is not possible to charge or replace their batteries. Therefore, energy efficient operation of wireless sensors to prolong the lifetime of overall wireless sensor network is of utmost importance [1,2]. Due to their low power radio, wireless sensor nodes cannot transmit the data to large distance in single hop, which makes multi-hop communication essential in case of real life deployment. However, in multi-hop cases, if the energy consumption of sensor nodes is not managed properly it may create energy-hole problem in the network [3]. In literature, a number of protocols have been proposed to manage and reduce the energy consumption of sensor nodes [1-8]. Grouping sensor nodes into clusters has been widely used to achieve this objective. In clustered networks, one of the sensor nodes is elected as cluster head for each cluster. Sensor nodes in each cluster transmit data to their respective cluster head and the cluster head in turn forwards the data after aggregation/fusion to sink node through single/multi-hop transmission.

LEACH is one of the most popular distributed single-hop clustering protocols [5]. In this protocol, the clusters are formed, based on received signal strength. The role of cluster head is periodically rotated amongst the sensor nodes present in the cluster to ensure balanced energy consumption of sensor nodes. This algorithm becomes very inefficient in case of large area sensor networks due to single hop communication of cluster heads to the sink. A number of improvements have been proposed in literature to overcome the shortcomings of LEACH [6, 15-17]. Some of them are LEACH-C [6], PEGASIS [16], TEEN [15], HEED [17], etc. Hausdorff [10] and ERP-SCDS (Energy Efficient Routing Protocol for wireless networks with Static Clustering and Dynamic Structure) [11] are recent clustering algorithms. In this paper, we propose an energy efficient protocol consisting of clustering, cluster head selection/rotation and data routing method to prolong the lifetime of sensor network. In proposed protocol, clusters are formed only once during the lifetime of sensor network, which results in substantial saving of energy.

The simulation experiments demonstrate that the proposed protocol substantially enhances the network lifetime of wireless sensor network. The rest of the paper is organized as follows: Section II describes the related work. Section III describes the system model, which includes network model and energy dissipation model for wireless sensor networks. Section IV describes proposed protocol, including formation of clusters, cluster head selection. Section V describes, energy cost calculation of cluster for its normal operation, and energy cost calculation for optimal cluster head selection/rotation process. Results of simulations for various performance metrics are 
given in Section VI. Concluding remarks have been incorporated in Section VII.

\section{RELATED WORK}

A number of clustering algorithms have been proposed for mobile ad hoc and wireless sensor networks [5-18]. Linked cluster algorithm (LCA) is one of the earliest works aimed towards prolonging network lifetime in mobile ad hoc networks $[18,19]$. LCA basically was focused towards forming an efficient network topology to handle the mobility of nodes. It was therefore geared for maximizing network connectivity. The LCA resulted in large number of clusters. Therefore, the algorithm was refined in [20]. Lin and Gerla proposed the efficient support of multimedia applications in multi-hop mobile networks using CDMA. Random competition based clustering (RCC), designed for mobile ad hoc networks can also be used for wireless sensor networks [21]. It mainly focuses at cluster stability in order to support mobile nodes. RCC is based on first declare first win rule. In this approach the first node declaring it as cluster head, forms a cluster within its radio range and rest of nodes in its radio coverage give up their right to become the cluster head and joins its cluster as members. CLUBS is an algorithm that forms cluster through local broadcast and coverage in a time proportional to the local density of nodes [22].

A multi-tier hierarchical control clustering algorithm was proposed by Banerjee and Khullar [23]. In this process, any node can initiate the process of cluster formation and clustering process is carried out in hierarchical order. Low Energy Adaptive Clustering Hierarchy (LEACH) is one of the most popular clustering algorithms for wireless sensor networks [5]. In LEACH clusters are formed based on the received signal strength. The sensor nodes make autonomous decision about cluster formation without any centralized control and cluster head nodes are used to route the data to the base station. The algorithm ensures the balanced energy usage of nodes by random rotation of cluster head role amongst the nodes inside cluster. LEACH forms one-hop intra and inter cluster topology, where each node transmits directly to the cluster head and after aggregation, the cluster head transmits the data to base station in single-hop. This is a serious drawback of LEACH, which makes it less efficient in real life for network deployed in large area. It is also less efficient if the cluster heads are far away from the sink. A number of algorithms have been proposed to overcome the shortcomings of LEACH, such as LEACH-C [6], TEEN [15], APTEEN [24], and PEGASIS [16], etc. Fast Local Clustering Service (FLOC) was proposed as a distributed technique that produces approximately balanced clusters with minimum overlap [25]. In Energy Efficient Clustering Scheme (EECS) the cluster head candidates compete amongst themselves based on the residual energy, for a given round and cluster head candidate with maximum residual energy get selected as cluster head [8]. EECS extends the LEACH algorithm by dynamic sizing of clusters based on the distance of cluster from the sink node/base station. This improves the balanced energy distribution throughout the network resulting in the extended lifetime of network. Hybrid Energy Distributed Clustering (HEED) is multi-hop clustering algorithm, where cluster heads are elected based on the residual energy and intracluster communication cost [17]. Intra-cluster communication cost is proportional to the node's proximity to its neighbor and is used by the node in deciding to join the network. It provide uniform cluster head distribution throughout the network and better load balancing. Energy-efficient Unequal Clustering (EEUC) addresses the hop-spot problem that exists due to the fact that cluster heads close to the base station die faster because they need to relay more data traffic [9]. EEUC solves this problem by balancing the energy consumption for forming unequal clusters, where cluster near the sink node are smaller in size than the clusters away from the sink node in order to save intra-cluster energy. Power Efficient and Adaptive Clustering Hierarchy (PEACH) cluster formation is based on overhearing characteristics of wireless communication to support adaptive multi-level clustering [26].

Hausdorff clustering algorithm is a static clustering method, in which the Hausdorff distance between two node set is used as clustering metric [10]. It carries out cluster formation based on minimum Hausdorff distance between nodes of same cluster and between the nodes of neighboring clusters, to ensure connectivity within the network. ERP-SCDS (Energy efficient routing protocol for wireless networks with static clustering and dynamic structure) utilizes virtual points in a corona based wireless sensor network, forming static clusters and dynamic structure [11]. This is a location based clustering protocol, where clustering process is carried out by virtual points around the sink. The location of virtual points is calculated by sink after receiving the location information from all sensor nodes in the network. The protocol involves the transmission of location information by all sensor nodes to the sink for initiation of clustering process. It increases the energy cost of clustering; specially, in case of large area sensor networks due to multi hop communication between nodes and sink.

\section{SYSTEM MODEL}

Wireless sensor network model based on circular monitoring area $\mathrm{A}$ of radius $\mathrm{Z}$ with uniform node distribution density $\rho$ having only one sink node at the center of monitoring area has been considered. There are $\mathrm{T}$ sensor nodes deployed in the sensing area, designated as $\mathrm{N}_{1}, \mathrm{~N}_{2}, \ldots . \mathrm{N}_{\mathrm{T}}$. Such model has been widely used in literature [6].

It is assumed that the transmitter electronics of all sensor nodes is capable of multi range transmission of the data with two types of radio ranges for intra cluster and inter cluster communication: low power broadcast range $R_{1}=R / 2$ meters and high power range of $R_{2}=R$ meters, where $R$ is the maximum transmission range of a node. Low power broadcast range $R_{1}$ is used for intra cluster communication between a sensor node and cluster head. The maximum diameter of any cluster is assumed to be $R / 2$ meters, so that even if a node is located at boundary of a cluster, it may be selected as cluster head, and data connectivity is still maintained between the sensor nodes and cluster head within the cluster.

High power broadcast rang $R_{2}$ is used by cluster head node to transmit data either to next hop cluster head or to the sink node, in case the sink node is at a distance of one hop from the cluster head. The proposed model ensures that two neighboring cluster head are always at maximum distance of $\mathrm{R}$ from each other, so that connectivity is always maintained between such nodes. The sensor nodes use radio range $\mathrm{R}_{3}=\mathrm{R} / 4$ during the 
clustering process in order to form clusters of balanced size. As the data from various sensor nodes arrive at the cluster head, it performs data aggregation or data fusion over the arrived data as per the nature of sensed data to reduce the transmission overhead. The cluster head transmits the aggregated/fused data to its next hop cluster head towards sink node. To avoid the collision of intra and inter cluster data; multiple access technique of TDMA and direct sequence spread spectrum (DS$\mathrm{SS})$ respectively is assumed [10]. All sensing nodes deployed in the sensing area are assumed to be static and have the knowledge of their location. The sensing field is divided into $\mathrm{m}$ numbers of concentric circles of equal width, known as corona [14]. In proposed model, the width of each corona is assumed to be $\mathrm{R} / 2$ meters, as illustrated in the Fig. 1 .

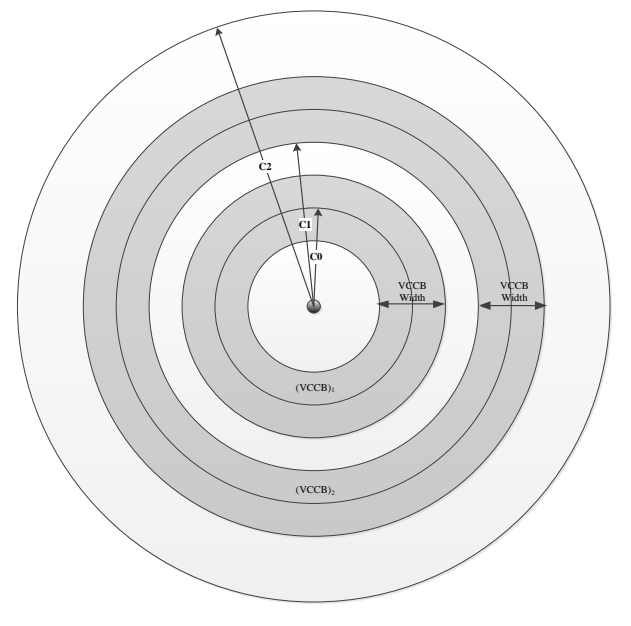

Figure 1. Corona based wireless sensor network and Assignment of concentric circles band (VCCB) to the sensor nodes.

\section{A. Energy Model}

Generalized energy consumption model based on first order radio energy consumption is used for calculation of energy consumption for sensor nodes within the sensing area $[5,6,12$, 13].The energy consumption of a sensor node for transmitting $\mathrm{k}$ bits of data over a distance $\mathrm{d}$ can be expressed as $[6,13]$ :

$$
\begin{aligned}
& E_{T x}(k, d)=E_{\text {elect }-T x}(k)+E_{\text {amp-Tx }}(k, d) \\
& E_{T x}(k, d)= \begin{cases}k E_{\text {elect }}+k \grave{o}_{f s} d^{2}, & d<d_{0} \\
k E_{\text {elect }}+k \grave{o}_{m p} d^{4}, & d \geq d_{0}\end{cases}
\end{aligned}
$$

$\mathrm{E}_{\text {elect-Tx }}$ is transmission electronics energy; which is energy consumed by the sensor node for modulation, coding, spreading schemes, filtering operations, etc. $E_{a m p-T x}(k, d)$ is the power amplifier stage energy consumption of sensor node to transmit $\mathrm{k}$ bits of data over a distance of $\mathrm{d}$ meter with acceptable signal to noise ratio $(\mathrm{SNR})$. $\mathrm{E}_{\text {elect }}(\mathrm{nJ} / \mathrm{bit})$ is energy dissipation per bit to run transmitter and receiver electronic circuitry. $\varepsilon_{\mathrm{fs}}\left(\mathrm{pJ} /\left(\mathrm{bit}_{\left.\left.-\mathrm{m}^{-2}\right)\right)}\right.\right.$ is energy coefficient of power amplifier stage of sensor node for free space energy dissipation model, when transmission distance is less than threshold i.e. $d$ $<\mathrm{d}_{0} . \varepsilon_{\mathrm{mp}}\left(\mathrm{pJ} /\left(\right.\right.$ bit- $\left.\left.^{-4}\right)\right)$ is energy coefficient of power amplifier stage of sensor node for multipath energy dissipation model, when transmission distance is greater than threshold i.e. $d \geq d_{0}$. The energy consumption of sensor node to receiver $k$ bits of data is given by:

$$
E_{R x}=k E_{\text {elect }}
$$

\section{Proposed Clustering Protocol}

The proposed protocol, Virtual Concentric Circle Band Based Clustering (VCCBC) is divided into several phases. After the deployment of sensor nodes in sensing area, the clustering and cluster head selection phase starts with transmission of beacon by sink node. The beacon contains information regarding the location of sink node $\left(\mathrm{x}_{\mathrm{s}}, \mathrm{y}_{\mathrm{s}}\right)$ within the network. After receiving the sink node location, all the sensor nodes calculate their respective Euclidian distance $\mathrm{d}_{\mathrm{si}}$, from the sink node. Once, all sensor nodes calculate their respective distance from the sink node, the formation of concentric circles around the sink node starts. In this process the sensor nodes are assigned concentric circle index $C_{j}(j=1$, $2,3, \ldots . \mathrm{m})$, each of width $\mathrm{R} / 2$. The assignment of concentric circle to the sensor node $\mathrm{N}_{\mathrm{i}}$ is carried out with the formula:

$$
C_{j}=\frac{2\left|d_{s i}\right|}{R}
$$

\section{A. Cluster Formation and Cluster Head Selection}

To achieve energy balanced clustering in the network, in proposed scheme, virtual concentric circles are designated as $\mathrm{V}_{1}, \mathrm{~V}_{2}, \mathrm{~V}_{3} \ldots$. A virtual concentric circle band lies at the midway between two concentric circles and has width $\pm \delta$ i.e. $2 \delta$. The index $\mathrm{V}_{\mathrm{j}}$ of virtual concentric bands can be calculated as:

$$
\begin{aligned}
& V_{j}=\left[\left\{\left(\frac{R}{2} * C_{i}\right)+\frac{R}{4}\right\} \pm \delta\right] \\
& \text { If } x_{i}=\left\{\left(\frac{R}{2} * C_{i}\right)+\frac{R}{4}\right\} \text { Then } V_{j}=\left[x_{i} \pm \delta\right]
\end{aligned}
$$

The information regarding the sensor node radio range $\mathrm{R}$ is known apriori to all nodes and value of $\delta$ is made known to all sensor nodes at the time of node deployment. The value of $\delta$ depends upon the node density in the network. All sensor nodes in the network calculate their respective VCCB index $V_{j}$ and compare it with their respective distance from the sink node $\mathrm{d}_{\mathrm{si}}$. If the distance $d_{s i}$, falls within the VCCB index $\left[x_{i} \pm \delta\right]$; the sensor node is declared as a probable candidate for the election of cluster head $(\mathrm{CH})$ in the first round. All other sensor nodes opt out from the process of cluster head selection for the first round and wait for the declaration of first round cluster head, to associate themselves with one of such declared cluster head. On completion of the process of selection of probable candidates for first round cluster head, the process of first round cluster head election starts. The best possible candidate for the election of first round cluster head would be the one located exactly midway between the two concentric circles. For the first round cluster head election, it has been assumed that the energy of all probable cluster head candidates is same. Therefore, only location of cluster head candidate has been 
used as sole criteria for first round cluster head selection. All the probable cluster head candidates calculate their distance from center of their respective VCCB as:

$$
d_{i(v c)}=\left|\left[\left\{\left(\frac{R}{2} * C_{i}\right)+\frac{R}{4}\right\}-d_{s i}\right]\right|
$$

Initially back-off timer value of node $\mathrm{N}_{\mathrm{i}}$ is set to be $\mathrm{t}_{\mathrm{i}}$, and is given by:

$$
t_{i}=\frac{d_{i(v c)}}{\delta} * T_{c h}
$$

Where $T_{\text {ch }}$ is the time allocated for the first round cluster head election. The sink node assists the cluster head formation by sending START message, which directs all the probable cluster head candidates in the network to start their back-off timer at same time. As the back-off timer value is directly proportional to the distance of cluster head candidate from center of VCCB, the back-off timer of the node near to the center will expire first. As soon as the back-off timer of particular cluster head candidate expires, it sends advertising message CH_ADVT, declaring itself as first round cluster head. This message will be transmitted within the radio range $\mathrm{R}_{3}=(\mathrm{R} / 4)$ of sensor node. Any other probable cluster head candidate within the radio range $(\mathrm{R} / 4)$ of the node which has declared itself as cluster head, will stop its back-off timer and associate itself with the declared cluster head for formation of cluster. Similarly all non-cluster head candidates, falling within the radio range $(\mathrm{R} / 4)$ of declared cluster head, will also associate themselves with the cluster head and thus form a uniform cluster. Same process takes place in all the concentric circles throughout the sensing area and simultaneous cluster head selection and cluster formation takes place within the network.

\section{Cluster Head Rotation}

The role of cluster head in a cluster must be rotated regularly amongst the sensor nodes to prolong the life time of sensor network by balancing the energy consumption of various sensor nodes. Since, cluster head is required to perform extra task of data gathering and data relaying, compared to the regular sensor nodes, its energy drains out faster. Therefore, some mechanism must be adopted to rotate the role of cluster head. The rotation mechanism must ensure balanced energy consumption of all the sensor nodes in cluster. A number of methods for cluster head rotation have been discussed in the literature [13-19]. Most of these methods are based on residual energy of cluster head or distance of prospective cluster head from the center of the cluster.

If uniform transmission power is assumed for intra cluster communication and cluster is assumed to be static, the distance parameter of elected cluster head from center of cluster becomes insignificant, since power consumption for communication remains same irrespective of the location of cluster head inside the cluster. For such cases, the residual energy of cluster head is important parameter to initiate the process of cluster head rotation. For our proposed model, the cluster head rotation process has been performed based on the residual energy of the cluster head, as explained in the next section. The frequency and timing of cluster head rotation process, aimed at optimum life time of wireless sensor network, is decided by calculating the energy consumption for regular sensor nodes and cluster head nodes for various tasks performed by these nodes including data relaying and cluster

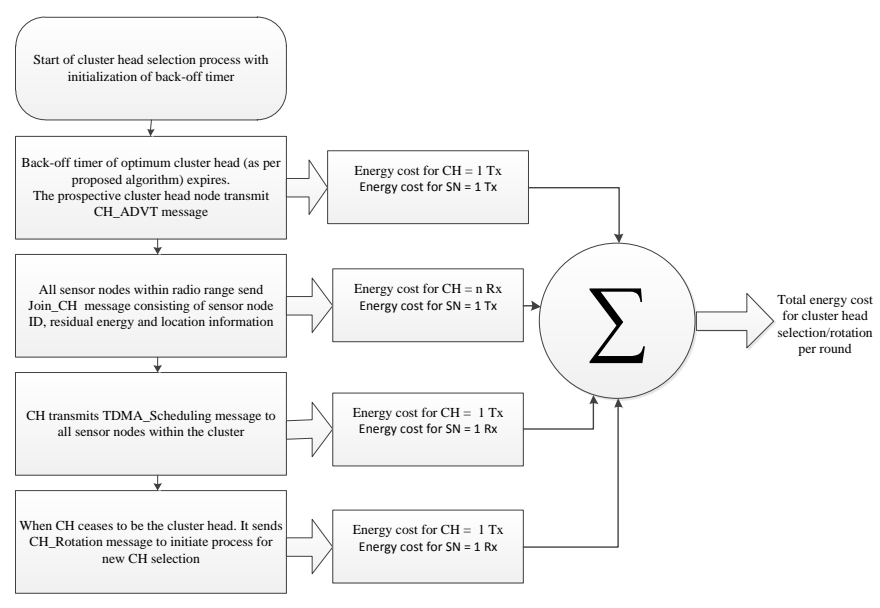

Figure 2. Total energy cost calculation for cluster head selection/rotation per round.

head selection/rotation. After assessing the actual energy consumption of sensor nodes, the balanced energy consumption is ensured by optimal rotation of cluster head node at regular intervals.

\section{A. Energy cost of sensor nodes for normal operation}

For normal operation of regular sensor node, energy consumption is basically attributed to the task of sensing, processing and communication of sensed data to the cluster head. Energy consumption of regular sensor node for normal operation can be expressed as:

$$
E_{S N}=\left[E_{\text {elect }}+E_{\text {sense }}+\in d_{S N-C H}^{n}\right] k
$$

Where, $\mathrm{E}_{\text {sense }}$ is the energy consumed by sensor node for sensing single bit of data and $\mathrm{d}_{\mathrm{SN}-\mathrm{CH}}$ is the distance of sensor node from its cluster head. If, there are n numbers of regular sensor nodes present in a cluster. Then, total energy consumed by all regular nodes in a cluster can be expressed as:

$$
E_{S N}=\left[E_{\text {elect }}+E_{\text {sense }}+\epsilon_{f s}\left(\frac{R}{2}\right)^{2}\right] n k
$$

The cluster head receives $\mathrm{k}$ bits data sensed by each node present in the cluster during one round of sensing. Apart from receiving the sensed data, cluster head also acts as a regular node to sense the area in its vicinity. It is also responsible for carrying out data aggregation or data fusion operation over the data received from all regular sensor nodes present in the cluster. Moreover, the cluster head is required to transmit data to next hop cluster head or sink node with maximum radio power which is good enough to transmit data over a distance R. Total energy consumption within the cluster for sensing $\mathrm{k}$ bits 
of data and transmitting this data towards sink node through single/multi-hop communication can be expressed as:

$$
\begin{gathered}
E_{\text {total (normal) })}=E_{S N}+E_{C H} \\
E_{\text {total (normal) }}=\left[E_{\text {elect }}+E_{\text {sense }}+\in_{f s}\left(\frac{R}{2}\right)^{2}\right] n k+n k E_{\text {elect }} \\
+k E_{\text {sense }}(n+1) k E_{\text {aggr }}+\left[E_{\text {elect }}+\in_{f s} R^{2}\right](n+1) \alpha k
\end{gathered}
$$

Where, $\mathrm{E}_{\mathrm{aggr}}$ is energy consumed for aggregating the data and $\alpha$ is the data aggregation coefficient. The sequence of messaging is shown in Fig. 2. Extra energy cost for normal sensor node during cluster head selection/rotation process has been calculated as per data transmission and reception carried out during one round of cluster head selection/rotation is given by:

$$
\begin{gathered}
E_{S N(\text { extra })}=1 T x_{(R / 2)}+3 R x \\
E_{S N(\text { extra })}=k\left[E_{\text {elect }}+\epsilon_{f s}\left(\frac{R}{2}\right)^{2}\right]+3 k E_{\text {elect }} \\
E_{S N(\text { extra })}=k\left[4 E_{\text {elect }}+\in_{f s}\left(\frac{R}{2}\right)^{2}\right]
\end{gathered}
$$

Since, there are $\mathrm{n}$ normal sensor nodes in the cluster, the total energy consumption of normal sensor nodes, per cluster head rotation process can be expressed as:

$$
E_{S N(\text { extra })}=n k\left[4 E_{\text {elect }}+\in_{f s}\left(\frac{R}{2}\right)^{2}\right]
$$

\section{B. Optimal Rotation of Cluster Head}

Optimal time/energy threshold to initiate the cluster head rotation process is calculated by analyzing the energy consumption of sensor node for regular sensing and cluster head selection/rotation process. Sensor energy calculation for regular and cluster head selection/rotation process has been analyzed in Section V A. In order to prolong the life time of the network, energy consumption of sensor nodes within the cluster has to be balanced. This balance can only be achieved if the role of a sensor node as cluster head is rotated at appropriate time/energy threshold. This threshold is calculated by assuming $n+1$ number of nodes in a cluster, out of which, $n$ nodes are assumed to act as regular sensing nodes and one sensor node in the cluster acts as cluster head. If energy of $\mathrm{N}_{\mathrm{i}}$ sensor node is assumed to be $\mathrm{E}_{\mathrm{i}}$, total energy of the cluster can be given as:

$$
E_{\text {total-cluster }}=\sum_{i=1}^{n+1} E_{i} \quad \text { Joules / cluster }
$$

Ideally, in a cluster based sensor network, the life of cluster can be prolonged when each sensor node in the network plays the role of cluster head at the most once during the whole life time of the network $[9,15]$. This ensures minimum energy consumption during the cluster head rotation and uniform energy drainage of sensor nodes. We have assumed $n+1$ number of sensor nodes in a cluster. Therefore, if every sensor node is given a chance to become cluster once during the life time of the sensor network; total number of cluster head rotations will be $n$. Energy cost of cluster for $n$ cluster head rotations is given as:

$$
E_{\text {total (extra-cluster })}=n\left[\begin{array}{l}
3 k\left\{E_{\text {elect }}+\in_{f s}\left(\frac{R}{2}\right)^{2}\right\}+\left(\begin{array}{l}
E_{\text {elect }}+ \\
\epsilon_{f s} R^{2}
\end{array}\right)+ \\
k n E_{\text {elect }}+n k\left\{4 E_{\text {elect }}+\in_{f s}\left(\frac{R}{2}\right)^{2}\right\}
\end{array}\right]
$$

Now, maximum number of possible rounds of data sensing and transmission (taking into consideration the additional energy cost of cluster head rotation/selection) will depend upon the total initial energy of cluster can be given by:

$$
\begin{gathered}
N_{\text {optimal }}=\frac{E_{\text {total-cluster }}-E_{\text {total (extra-cluster })}}{E_{\text {total (normal })}} \\
N_{\text {optimal }}=\frac{\left[(n+1) E-n\left[3 k\left\{E_{\text {elect }}+\epsilon_{f s}\left(\frac{R}{2}\right)^{2}\right\}+\left(\begin{array}{l}
E_{\text {elect }}+ \\
\epsilon_{f s} R^{2}
\end{array}\right)+k n E_{\text {elect }}+n k\left\{4 E_{\text {elect }}+\epsilon_{s s}\left(\frac{R}{2}\right)^{2}\right\}\right]\right]}{\left[E_{\text {elect }}+E_{\text {sense }}+\epsilon_{s s}\left(\frac{R}{2}\right)^{2}\right] n k+n k E_{\text {elect }}+k E_{\text {sense }}(n+1) k E_{\text {agse }}+\left[E_{\text {elect }}+\in_{f s} R^{2}\right](n+1) \alpha k}
\end{gathered}
$$

For minimal energy cost of cluster head rotation, the cluster head rotation must take place uniformly after $\left[\left(\mathrm{N}_{\text {optimal }}\right) / \mathrm{n}\right]$ rounds. In the proposed model, cluster head rotation process is carried out based on the number of sensing rounds for cluster head. The cluster head in each cluster keeps on counting the number of sensing rounds, it had performed, and after each $\left[\left(\mathrm{N}_{\text {optimal }}\right) / \mathrm{n}\right]$ rounds, it initiates the process of cluster head rotation and after completion of the cluster head rotation process next sensor node start acting as cluster head in the cluster. As $\left[\left(\mathrm{N}_{\text {optimal }}\right) / \mathrm{n}\right]$ rounds are calculated based on the optimized energy threshold; proper energy balance is achieved to prolong the life of sensor network. However, in our model, the cluster head, instead of tracking its residual energy only uses simple counter to count the number of sensing rounds.

\section{PERFORMANCE EVALUATION}

In this section the performance of VCCBC protocol is evaluated through simulation experiments. We have implemented the simulator in MATLAB. The performance of VCCBC protocol is compared with Hausdorff [10] and ERPSCDS [11] protocols.

\section{TABLE I. SIMULATION PARAMETERS}

\begin{tabular}{|l|l|l|}
\hline \multicolumn{1}{|c|}{ Parameter } & \multicolumn{1}{c|}{ Default Value } & \multicolumn{1}{c|}{ Range } \\
\hline Area & $(100 \times 100) \mathrm{m}^{2}$ & $(50 \times 50) \sim(400 \times 400) \mathrm{m}^{2}$ \\
\hline Number of nodes & 400 & $100 \sim 500$ \\
\hline Initial Energy of node & 2 Joule & \\
\hline Data packet size $(\mathrm{k})$ & $100 \mathrm{byte}$ & \\
\hline $\mathrm{E}_{\text {elect }}$ & $50 \mathrm{~nJ} / \mathrm{bit}$ & \\
\hline$\varepsilon_{\mathrm{fs}}$ & $10 \mathrm{pJ} / \mathrm{bit} / \mathrm{m}^{2}$ & \\
\hline $\mathrm{E}_{\mathrm{mp}}$ & $0.00134 \mathrm{pJ} / \mathrm{bit} / \mathrm{m}^{4}$ & \\
\hline $\mathrm{E}_{\mathrm{aggr}}$ & $5 \mathrm{~nJ} / \mathrm{bit} / \mathrm{signal}$ & \\
\hline Threshold distance $\left(\mathrm{d}_{0}\right)$ & $87 \mathrm{~meters}$ & \\
\hline
\end{tabular}


The performance metrics include clustering energy dissipation, and life-time of network. The probability of signal collision and signal interference is ignored, assuming TDMA scheduling for intra cluster and DS-SS for inter cluster communication. The simulation parameters are listed in Table I. For each parameter, simulation has been run many times and average result of all runs has been taken for evaluation.

\section{A. Clustering Energy Dissipation}

Minimum clustering energy requirement can prolong the network lifetime and can be used as parameter to demonstrate the efficiency of any clustering and routing protocol. Fig. 3 shows the variation in clustering energy dissipation with variation in number of nodes and area of sensing field. Fig. 3(a) shows the simulation results of variation in clustering energy dissipation, in case of Hausdorff, ERP-SCDS and VCCBC protocol with variation in number of nodes from 100 to 500 , when nodes are randomly deployed in a sensing field of $100 \times 100 \mathrm{~m}^{2}$. The clustering energy dissipation is highest in case of Hausdorff, because it uses more control messages for the formation of clusters. ERP-SCDS requires less energy for clustering than Hausdorff protocol, yet its clustering energy requirement is more than VCCBC protocol. Since, it requires the location information transmission of every node to the sink and after receiving this information, the sink initiates the clustering process, dissipating more energy than our proposed protocol VCCBC. This process involves large number of transmit/receive operations, increasing the clustering energy cost. In case of VCCBC protocol, first round cluster head selection and clustering is carried out simultaneously throughout the network. It uses less number of transmit/receive operations since, less number of control messages are exchanged for clustering. Fig. 3(b) shows the simulation results of variation in the clustering energy dissipation with increase in sensing area side length from 50 to 400 meters, when 400 numbers of nodes are deployed. The simulation results show the increase in clustering energy dissipation for Hausdorff and ERP-SCDS. This increase can be attributed to the exchange of more control messages in case of Hausdorff and more transmission of location information from all sensor nodes to the sink with increase in distance of nodes from the sink, in case of ERP-SCDS.

\section{B. Network lifetime}

Network lifetime of wireless sensor network is the time span from the deployment to the instant the network ceases to achieve objectives of its deployment. We have considered number of rounds till first node dies (FND), as parameter for the evaluation of VCCBC protocol and existing protocols. Fig. 3 shows simulation results of variation in network life time, i.e. total number of rounds from the deployment to the instant when first node dies in the network. In Fig. 4(a) the number of rounds has been plotted against number of sensor nodes varying from 100 to 500 deployed in sensing field of $100 \times 100$ $\mathrm{m}^{2}$. The simulation results show that VCCBC protocol exhibits better lifetime than Hausdorff and ERP-SCDS protocols. In case of Hausdorff, the unbalanced clustering and more overhead messages for clustering are responsible for the lower lifetime. ERP-SCDS has better network lifetime than Hausdorff due to formation of balanced clusters, yet clustering requires more energy, causing less lifetime of network in comparison to proposed protocol. Proposed protocol shows

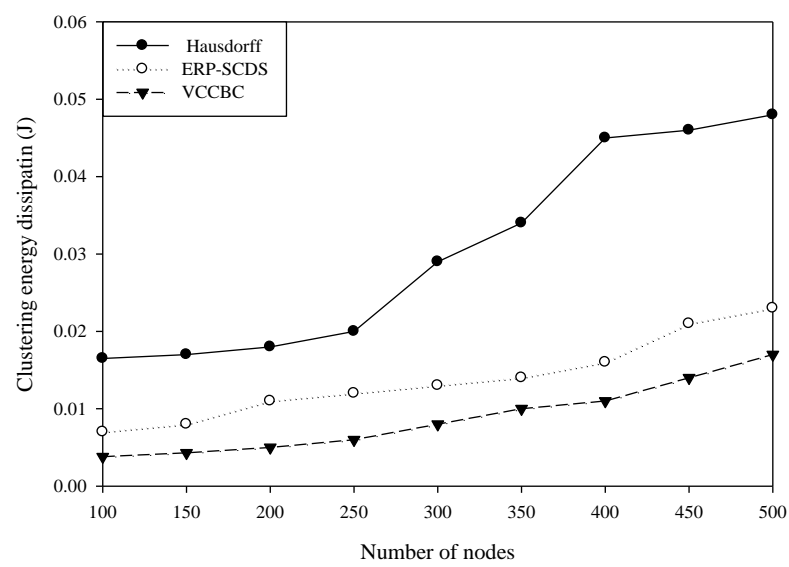

(a)

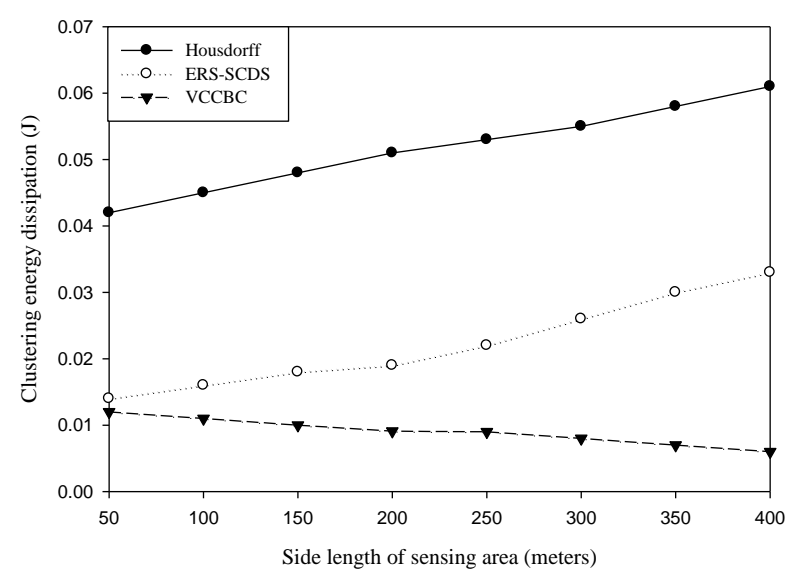

(b)

Figure 3. Variation in Clustering energy dissipation with (a) number of nodes deployed in sensing field, and (b) side length of sensing field.

highest network lifetime among these protocols, because, it require less energy for clustering, the cluster formed are well balanced and cluster head rotation is carried out considering all kinds of energy consumption within the network, providing more balanced energy depletion of sensor nodes in the cluster. Fig. 4(b) shows the simulation results of variation in network lifetime with increase in side length of sensing field from 50 to 400 , keeping the node deployment fixed to 400. Proposed VCCBC protocol exhibits better network life time in comparison to ERP-SCDS and Hausdorff protocols, despite increase in network area. This is due to the fact that in case of VCCBC protocol, the percentage increase in nodes acting as cluster head, decrease in number of nodes per cluster, and increase in percentage of single node clusters is smaller in comparison to these protocols.

\section{CONCLUSIONS}

An energy efficient clustering, cluster head selection/rotation and data routing protocol is proposed in this 
paper. The proposed virtual concentric circle band based clustering protocol (VCCBC) ensures the formation of uniform clusters in

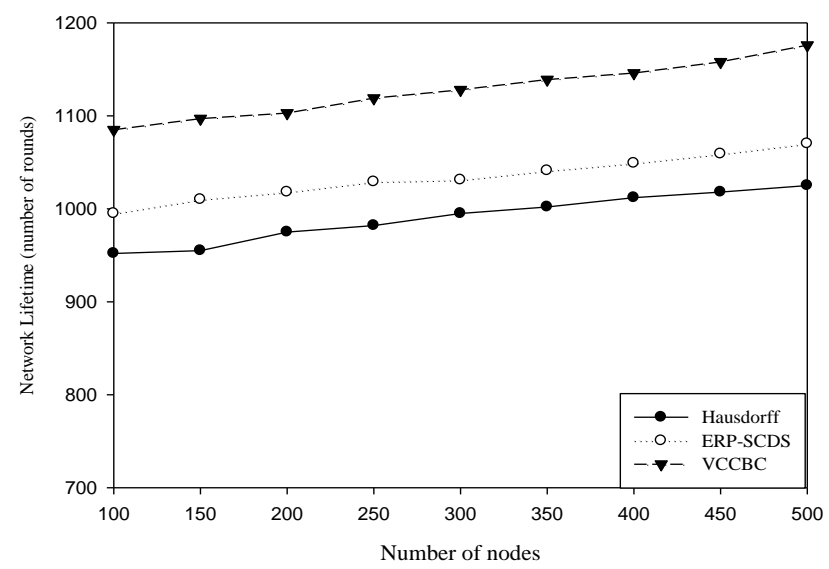

(a)

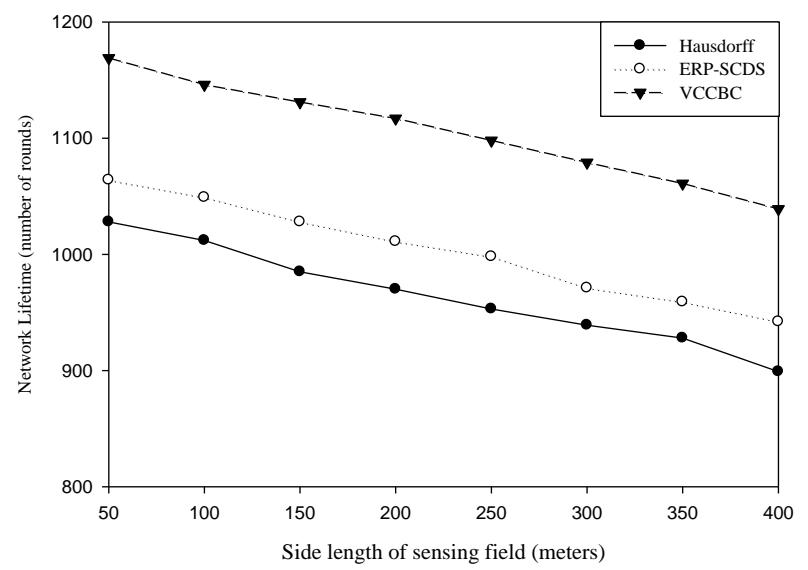

(b)

Figure 4. Variation in network lifetime with (a) number of nodes deployed in sensing field, and (b) side length of sensing field.

virtual concentric circular bands around the sink. In proposed method the cluster formation takes place only once in network lifetime, thus avoiding energy wastage associated with reclustering process. The cluster head rotation process is based on energy calculation of various tasks performed by the sensor nodes and cluster head in a cluster. The timing and frequency of cluster head rotation is carried out by balancing the energy consumption. This results in balanced energy drainage of the node in network, improving the network lifetime. The simulation results demonstrate the effectiveness of proposed protocol in term of clustering energy dissipation.

\section{REFERENCES}

[1] C.Y. Chong and S.P. Kumar, "Sensor networks: Evolution, Opportunities, and Challenges," Proceedings of the IEEE, vol. 91, no. 8, pp. 1247-1256, August 2003.

[2] Ameer Ahmed Abbasi and Mohamed Younis, "A Survey on Clustering Algorithms for Wireless Sensor Networks," Elsevier Journal of Computer Communications, vol. 30, pp. 2826-2841, October 2007.
[3] Xiaobing Wu, Guihai Chen and S.K. Das, "Avoiding Energy Holes in Wireless Sensor Networks with Nonuniform Node Distribution," IEEE Transactions on Parallel and Distributed Systems, vol. 19, no. 5, pp. 710-720, May 2008.

[4] M. Younis, M. Youssef and K. Arisha, "Energy-Aware Management in cluster-Based Sensor Networks," International Journal on Computer Networks, vol.43, no. 5, pp. 649-668, December 2003.

[5] W. Heinzelman, A. Chandrakasan and H. Balakrishnan, "EnergyEfficient Communication Protocol for Wireless Micro Sensor Networks" Proceedings of the 33rd Hawaii International Conference on System Sciences (HICSS '00), vol.2, pp. 234-243, January 2000.

[6] Wendi B. Heinzelman, Anantha P. Chandrakasan and Hari Balakrishnan, "An Application-Specific Protocol Architecture for Wireless Microsensor Networks", Transactions on Wireless Communications, Vol. 4, pp.660-670, October 2002.

[7] An Amir Sepasi Zahmati, Bahman Abolhassani, Ali Asghar Beheshti Shirazi, and Ali Shojaee Bakhtiari, "Energy-Efficient Protocol with Static Clustering for Wireless Sensor Networks," World Academy of Science, Engineering and Technology vol. 28, pp. 69-72, 2007.

[8] M. Ye, C. Li, G. Chen and J. Wu, "An Energy Efficient Clustering Scheme in Wireless Sensor Networks," Journal of Ad Hoc \& Sensor Wireless Networks, Vol.1, pp. 1-21, 2006.

[9] C. Li, M. Ye, G. Chen and J. Wu, "An Energy-Efficient Unequal Clustering Mechanism for Wireless Sensor Networks," Proceedings of IEEE International Conference on Mobile Ad hoc and Sensor Systems Conference(MASS05), Washington, D.C., pp. 604-611, November 2005.

[10] Xiaorong Zhu, Lianfeng Shen and Tak Shing Peter Yum, "Hausdorff Clustering and Minimum Energy Routing for Wireless Sensor Networks," IEEE Transaction on Vehicular Technology, vol. 58, no.2, pp. 990-997, February 2009.

[11] Huei Wen Ferng, Robby Tendean and Arief Kurniawan, "Energy Efficient Routing Protocol for Wireless Networks with Static Clustering and Dynamic Structure," Springer Journal of wireless personal communication (published online), March 2001.

[12] D. Goldenberg, A. Krishnamurthy, W. Maness, R. Yang, A. Young, A.S. Morse, A. Savvides and B.D.O. Anderson, "Network localization in partially localizable networks," IEEE INFOCOM, vol. 1, pp. 313-326, 2005.

[13] I. Rekleitis, D. Meger and G. Dudek, "Simultaneous Planning, Localization, and Mapping in a Camera Sensor Network," Robotics and Autonomous Systems, vol. 54, no.11, pp.921-932, 2006.

[14] Haibo Zhang and Hong Shen, "Balancing Energy Consumption to Maximize Network Lifetime in Data-Gathering Sensor Networks," IEEE Transactions on Parallel and Distributed Systems, vol. 20, no.10, pp.1526-1539, October 2009.

[15] A. Manjeshwar and D. Agrawal, "TEEN: A Routing Protocol for Enhanced Efficiency in Wireless Sensor Networks," Proceedings of 15th International Parallel and Distributed Processing Symposium (IPDPS'01), San Francisco, CA, pp. 2009-2015, April 2001.

[16] S. Lindsey and C. Raghavendra, "PEGASIS: Power-Efficient Gathering in Sensor Information Systems," Proceedings of IEEE Aerospace Conference, Big Sky, Montana, USA, vol.3, pp.1125-1130, March 2002.

[17] O. Younis and S. Fahmy, "HEED: A Hybrid Energy-Efficient Distributed Clustering Approach for Ad Hoc Sensor Networks," IEEE Transactions on Mobile Computing, vol. 3, no.4, 2004.

[18] D.J Baker and A. Ephremides, "The Architectural Organization of a Mobile Radio Network via a Distributed Algorithm," IEEE Transactions on Communications, vo. 29, no.11, pp. 1694-1701, 1981.

[19] D.J Baker and A. Ephremides, "The Design and Simulation of a Mobile Radio Network with Distributed Control," IEEE Journal on Selected Areas in Communications, vol. 2, no.1, pp. 226-237, January 1984.

[20] A. Ephremides, J.E. Wieselthier and D.J. Baker, "A Design Concept for Reliable Mobile Radio Networks with Frequency Hopping Signaling," Proceedings of IEEE vol. 75, no.1, pp.56-73, January 1987.

[21] K.Xu and M. Gerla, "A Heterogeneous Routing Protocol Based on A New Stable Clustering Scheme," Proceeding of IEEE Military Communications Conference, Anaheim, CA, pp. 123-131, October 2002. 
[22] R. Nagpal and D. Coore, "An Algorithm for Group Formation in an Amorphous Computers," Proceedings of 10th International Conference on Parallel and Distributed Systems (PDCS'98), Las Vegas, NV, October 1998.

[23] M. Younis, K. Akkaya and A. Kunjithapatham, "Optimization of Task Allocation in A Cluster-Based Sensor Network", Proceedings of the 8th IEEE Symposium on Computers and Communications (ISCC'2003), Antalya, Turkey, June 2003.

[24] A. Manjeshwar and D. Agrawal, "APTEEN: A Hybrid Protocol for Efficient Routing and Comprehensive Information Retrieval in Wireless Sensor Networks," Proceedings of International Parallel and Distributed Processing Symposium (IPDPS'02), Fort Lauderdale, Florida, pp.195202, April 2002.

[25] M. Demirbas, A. Arora and V. Mittal, "FLOC: A Fast Local Clustering Service for Wireless Sensor Networks," Proceedings of Workshop on Dependability Issues in Wireless Ad Hoc Networks and Sensor Networks (DIWANS'04), Palazzo dei Congressi, Florence, Italy, June 2004.

[26] S. Yi, J. Heo, Y. Cho, and J. Hong, "PEACH: Power-Efficient and Adaptive Clustering Hierarchy Protocol for Wireless Sensor Networks," Elsevier Journal on Computer Communications, vol. 30, pp. 2842-2852, October 2007

\section{AUTHORS PROFILE}

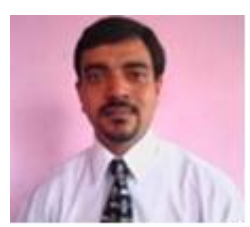

Ashok Kumar is working as Associate Professor in the Department of Electronics and Communication Engineering, National Institute of Technology Hamirpur Himachal Pradesh- INDIA. Presently he is pursuing PhD from National Institute of Technology. His current research areas of interest include wireless communication and wireless sensor networks. He has published more than 20 research papers in International/National journals and conferences in these areas. He is life mem member of ISTE.

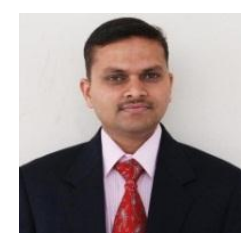

Dr. Narottam Chand received his $\mathrm{PhD}$ degree from IIT Roorkee in Computer Science and Engineering. Previously he received M Tech and B Tech degrees in Computer Science and Engineering from IIT Delhi and NIT Hamirpur respectively. Presently he is working as Associate Professor, Department of Computer Science and Engineering, NIT Hamirpur. He has served as Head, Department of Computer Science \& Engineering, during Feb 2008 to Jan 2011 and Head, Institute Computer Centre, during Feb 2008 to July 2009. He has coordinated different key assignments at NIT Hamirpur like Campus Wide Networking, Institute Web Site, Institute Office Automation. His current research areas of interest include mobile computing, mobile ad hoc networks and wireless sensor networks. He has published more than 120 research papers in International/National journals \& conferences and guiding six PhDs in these areas. He is member of IEEE, ISTE, CSI, International Association of Engineers and Internet Society.

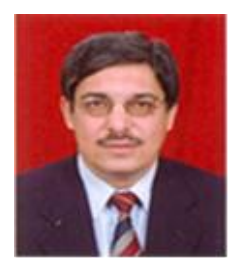

Dr. Vinod Kapoor was born at Mandi town in Himachal Pradesh, India. He received his $\mathrm{BE}$ Degree in Electronics \& Communication Engineering from National Institute of Technology (formerly Regional Engineering College), Durgapur, West Bengal, in the year 1987 and Masters Degree in Electronics \& Control from Birla Institute of Technology \& Science, Pilani (Rajasthan) in the year 1996. He did his Ph. D. from Kurukshetra University, Kurukshetra in the field of Optical Fiber Communication in Sept. 2006. He also obtained his MBA degree with specialization in Human Resources Management in the year 2002.He is presently Professor in the Department of Electronics \& Communication Engineering NIT Hamirpur, India. $\mathrm{He}$ has coordinated different key assignments at NIT Hamirpur like Training \& Placement Officer, Chief Warden (Hostels), organizing short term courses. His research interest includes optical fiber communication and optoelectronics/nano devices. He has published more than 30 research papers in International/National journal \& conferences and guiding four $\mathrm{Ph} . \mathrm{Ds}$ in these areas. He is member of ISTE, IETE and

IE. 